\title{
Can Asian Elephants Use Water as a Tool in the Floating Object Task?
}

\author{
Lisa P. Barrett ${ }^{1,2^{*}}$ and Sarah Benson-Amram ${ }^{1,2}$
}

\author{
${ }^{1}$ Zoology and Physiology, University of Wyoming \\ ${ }^{2}$ Program in Ecology, University of Wyoming \\ ORCID ID: Lisa P. Barrett - 0000-0001-6072-3479, Sarah Benson-Amram - 0000-0003-0147-7559 \\ *Corresponding author (Email: Lisapbarrett.Lpb@gmail.com)
}

Citation - Barrett, L. P., \& Benson-Amram, S. (2020). Can Asian elephants use water as a tool in the floating object task? Animal Behavior and Cognition, 7(3), 310-326. doi: https://doi.org/10.26451/abc.07.03.04.2020

\begin{abstract}
One of the greatest challenges in comparative cognition is to design tasks that accurately assess cognitive abilities across a diverse set of taxa with differing morphologies and behaviors. The floating object task was designed to test insightful problem solving via water tool use in animals but so far has been tested only in primates. In the floating object task, animals add water to a tube in order to reach a floating food reward. A similar task, the Aesop's fable task, which is solved by adding stones to the tube, has been used with corvids and raccoons in addition to human children. Elephants are considered to exhibit complex cognitive abilities on par with primates, and they possess a prehensile trunk appendage well-suited for tests of water tool use. Here, we presented the floating object task to 12 zoo-housed Asian elephants (Elephas maximus) to determine if they demonstrate innovative problem solving or social learning. One elephant solved the task on her own. Additionally, elephants at one zoo that observed a conspecific solve the task exhibited increased interest in the task compared to baseline elephants, demonstrating social learning via stimulus enhancement. Asian elephants are capable of learning to use water as a tool, but the cognitive abilities underpinning their ability to solve the floating object task remain unclear. Our findings may bolster support for the convergent cognitive evolution of problem solving in elephants and apes, but further research using additional paradigms is needed.
\end{abstract}

Keywords - Causal understanding, Elephas maximus, Innovation, Insight, Problem solving, Stimulus enhancement

The field of comparative cognition relies on leveraging standardized paradigms for inter-species comparison to elucidate the evolution of cognitive abilities, such as innovation, tool use, and social learning. The floating object task was originally developed to measure insightful problem solving - the sudden production of a new response without trial and error (Thorpe, 1963) (i.e., an "ah-ha" moment) by using water as a tool to bring a floating food reward within reach (Mendes et al., 2007). Innovative tool use is a complex behavior because it requires an understanding about how to use an external item in a creative way (reviewed in St. Amant \& Horton, 2008). Despite great interest in tool use and insight, it has been challenging to study these abilities in a range of species due to how infrequently insight and tool use are observed; moreover, researchers have struggled to demonstrate insightful problem solving, because it is difficult to test for an "ah-ha" moment in nonhuman animals (Shettleworth, 2012).

Since Povinelli's writing of Folk Physics for Apes, the field of comparative cognition has developed new tests of insightful learning (Povinelli, 2000). Insight tasks like the floating object task have now been used with several primate species (capuchin monkeys, Cebus apella or Sapajus apella, 
Renner et al., 2017; orangutans, Pongo abelii, Mendes et al., 2007; chimpanzees, Pan troglodytes, Tennie et al., 2010; Bornean orangutans, Pongo pygmaeus, gorillas, Gorilla, chimpanzees, humans, Homo sapiens, Hanus et al., 2011), and the Aesop's Fable task (a variant of the floating object task in which subjects drop stones to raise the water level) has been used with mainly corvid species, Corvus spp. (e.g., Bird \& Emery, 2009a, b; Jelbert et al., 2014; Logan, 2016; Logan et al., 2014; Taylor et al., 2011; but see an attempt with giant river otters, Pteronura brasiliensis, Asian small-clawed otters, Aonyx cinereus, and North American river otters, Lontra canadensis, Gormley, 2015). The results of these studies demonstrate that orangutans, chimpanzees, gorillas, humans, and several corvid species are capable of insightful learning and tool use. But other taxa that rely on non-visual sensory modalities and possess unique or highly dexterous morphology have rarely been tested. For example, when the Aesop's Fable task was recently presented to raccoons, Procyon lotor, the dexterous animals interacted with the task in novel and unexpected ways, such as solving the task by using floating objects to splash the marshmallow reward up the sides of the tube, and by continuing to add stones to the tube after retrieving a reward (Stanton et al., 2017). The untraditional ways in which the raccoons solved the Aesop's task, and their failure to preferentially select the predicted objects in some of the subsequent choice trials, was likely due to their attraction to handling objects and not due to a lack of understanding about the task. These findings suggest that other untested taxa may require further considerations (e.g., how sensory modalities play a role in interactions with the task) for these types of paradigms.

Elephants, Loxodonta spp. and Elephas maximus, are highly social, possess a dexterous and prehensile trunk, and exhibit complex behavior (body-self awareness: Dale \& Plotnik, 2017; insightful problem solving: Foerder et al., 2011; social learning: Greco et al., 2013; tool-use: Hart \& Hart, 1994; Hart et al., 2001; Mizuno et al., 2016; Wickler \& Seibt, 1997; means-to-end problem solving: Highfill et al., 2016; Irie-Sugimoto et al., 2008; acoustic recognition and discrimination: McComb et al., 2000, 2014; odor discrimination: Bates et al., 2007 human cue discrimination: Polla et al., 2018; odor and color classification: Bates et al., 2007; empathy: Plotnik \& de Waal, 2014a; olfactory quantity discrimination: Plotnik et al., 2019; habituation: Goodyear \& Schulte, 2015; cooperation: Plotnik et al., 2011; selfrecognition: Plotnik et al., 2006), but evidence of causal understanding in this taxon is equivocal. A lidremoval study in Asian elephants, Elephas maximus, tested whether elephants would still touch a lid even when it was not covering a food reward and concluded that elephants do not demonstrate causal understanding due to their functional fixedness (Nissani, 2006), which is the inability to use an object/tool for a new function after learning to use it for a different function (Duncker, 1945). For example, if Asian elephants demonstrate functional fixedness in the floating object task, they would view water only as a means of satiating thirst and not as a tool that can be used to bring a floating object within reach. We note, however, that the behaviors observed in the lid-removal study were likely due to routinized behavior (i.e., continuing to do a behavior even when a reward is available) instead of functional fixedness (Veling \& Aarts, 2012) due to the elephants being rewarded for touching a lid during a pre-training phase (Brown, 2018; Plotnik et al., 2010). Moreover, Nissani's (2006) claim that elephants demonstrate functional fixedness is surprising given elephants' tendency to use tools, such as sticks and water, for other reasons besides eating, such as grooming and bathing (Hart et al., 2001; Kurt \& Hartl, 1995; McKay, 1973; Moss, 1988). Although functional fixedness and insightful problem solving do not necessarily contradict each other, since problem-solving tasks do not always require using an item in a novel way, it also appears unlikely that elephants exhibit high levels of functional fixedness given the findings of a previous study in which Asian elephants demonstrated insightful problem solving (i.e., they were able to overcome any functional fixedness) by moving a block toy, which previously had never been used to acquire food, to stand on it to reach hanging food (Foerder et al., 2011).

Elephants represent the largest extant animals on land and rely more on olfactory and acoustic information than on visual information (Bates et al., 2007; Bates, Sayialel et al., 2008; McComb et al., 2000, 2014; Plotnik et al., 2014; Plotnik \& de Waal, 2014b), which makes designing and modifying tasks to assess cognitive abilities in elephants challenging, especially when these tasks were originally developed for other species. Elephant visual acuity is understudied, but generally thought to be moderate (i.e., maximal acuity of 13.16-14.37 cycles/degree - about 4.5 times less than human visual acuity, 
reviewed in Pettigrew et al., 2010), with dichromatic (Yokoyama et al., 2005) and most likely alternating monocular and binocular vision (Stone \& Halasz, 1989). They also have a relatively underdeveloped occipital lobe (Shoshani et al., 2006). The floating object task, in particular, has been tested with only highly visual species (i.e., primates). In order to know whether the floating object task is effective as a cross-species paradigm, however, and to better understand the evolution of insightful problem solving and tool use, we need to expand beyond these species to more diverse taxa, including those that are understudied or difficult to test empirically. By working with Asian elephants, we tested the classic floating object paradigm in a species that relies more on olfactory and auditory cues than on visual cues, and that navigates the environment with a prehensile trunk instead of with a beak or hands/paws.

In addition to using the floating object task to assess individual insightful tool use and problem solving in Asian elephants, we followed recent studies that have expanded on the traditional application of this task to determine whether it could also be used to assess social learning abilities (Nielsen, 2013; Renner et al., 2017; Tennie et al., 2010). Despite elephants' complex social structure and extensive communication, social learning in elephants has rarely been documented aside from anecdotes (Byrne et al., 2009). One experimental study in African bush elephants, Loxodonta africana, found evidence of social facilitation on a novel foraging task in captivity (Greco et al., 2013). Social facilitation occurs when the presence of a demonstrator affects an observer's behavior (Hoppitt \& Laland, 2013; Zajonc, 1965). The social facilitation found in Greco et al. (2013)'s study potentially occurred through localized stimulus enhancement, during which an observer is more likely to visit or interact with objects at a specific location after a demonstrator interacts with objects at that particular location (Greco et al., 2013; Thorpe, 1963). Here, we examined whether Asian elephants would learn how to solve the floating object task after watching a conspecific solve the task, either through stimulus enhancement or by copying the observed solution to the task via a form of social learning called imitation (i.e., after observing a demonstrator perform a novel action an observer becomes more likely to perform the same action) or emulation (i.e., an observer is more likely to produce the same result as that of the demonstrator but not necessarily by using the same action). These social learning mechanisms are not mutually exclusive, and it is possible that elephants can also learn the solution to the problem through multiple social learning mechanisms (Hoppitt \& Laland, 2013). To adapt the task to assess social learning abilities in elephants, we used a demonstrator-observer-control setup (Hoppitt \& Laland, 2013), where solvers served as demonstrators to conspecifics (i.e., observers) and control (i.e., baseline) elephants did not receive a demonstration. Observers were tested individually without the presence of the demonstrator, and we were therefore unable to assess social facilitation in this study.

Due to elephants' complex social behaviors exhibited in wild (e.g., keeping track of herd members: Bates, Sayialel et al., 2008; fission-fusion grouping: de Silva et al., 2011; matriarch leadership: McComb et al., 2001, 2011), and captive (e.g., cooperation: Plotnik et al., 2011; social learning: Greco et al., 2013; empathy: Plotnik \& de Waal, 2014a) settings, we expected that observers that received a demonstration from a conspecific would show evidence of social learning (e.g., via imitation, emulation, or stimulus enhancement) using visual and/or olfactory and/or auditory cues, whereas we expected baseline elephants would have lower success rates or take longer to solve compared to observers. In particular, we predicted that, if observers learn via localized stimulus enhancement, then they would exhibit increased persistence (i.e., greater proportion of time spent interacting with the task), and decreased neophobia toward the task (i.e., latency to touch the task, Benson-Amram et al., 2014; BensonAmram \& Holekamp, 2012) after observing the behaviors needed to solve the task, compared to baseline elephants. We predicted that elephants that learn through imitation or emulation would solve the task faster than baseline elephants. If elephants learn through imitation, we expected observers to show behavior patterns similar to those of their demonstrator. If they learn through emulation, we expected observers would show behavior patterns different from those of their demonstrator and learn to solve the task faster than baseline elephants.

Given elephants' use of their trunk to suck and dispense water (Kier \& Smith, 1985; Smith \& Kier, 1989), use of tools (Hart \& Hart, 1994; Hart et al., 2001; Mizuno et al., 2016; Wickler \& Seibt, 1997), and demonstration of insightful problem solving (Foerder et al., 2011), we expected that elephants 
would be capable of solving the task. If an elephant solved via insight we predicted that their latency to solve, latency to first add water (Mendes et al., 2007), and exploratory diversity would drop off after their initial interaction with the task such that they would demonstrate near-perfect performance on the task (Foerder et al., 2011; Shettleworth, 2012; Thorpe, 1963) (i.e., we would see a sharp decrease in latencies and exploratory diversity). In contrast, if elephants solved via trial-and-error learning, we expected to see a more gradual decrease in latency to solve, latency to first add water, and exploratory diversity (BensonAmram \& Holekamp, 2012; Daniels et al., 2019; Shettleworth, 2010).

Method

\section{Subjects}

Subjects were 12 Asian elephants housed at Smithsonian's National Zoological Park (NZP) and the Oklahoma City Zoo (OKC) that ranged in age from two to 69 years (Table 1), housed in accordance with AZA guidelines and approved by NZP (IACUC\# NZP 15-25) and OKC (IACUC\# 2015-013). Subjects were in good overall health and expected to be physically able to see the apparatus and demonstrations from other elephants, according to keepers. Subjects were not familiar with the apparatus prior to testing. One female elephant, Shanthi (NZP), had been known to add water to enrichment items, such as foraging boxes containing food pellets, but this behavior had not been studied empirically. Kandula (OKC) had also been known to use water for behaviors other than drinking and bathing, such as taking it from a pond and pouring it onto a manhole cover in an outdoor exhibit. The elephants were also familiar with sucking up and spitting out water for routine tuberculosis testing (i.e., "trunk washes"). Shanthi (NZP), Ambika (NZP), and Kandula (OKC) participated in a previous study of insightful problem solving where Kandula accessed an out-of-reach food reward by moving and standing on a block beneath the food (Foerder et al., 2011); however, the task used in that study is unrelated to the floating object task. Subjects were not food-deprived before being tested alone in an indoor enclosure. Testing enclosures contained only one automatic-refilling water drinker approximately $30.5 \mathrm{~cm}$ from the apparatus at NZP and $91.44 \mathrm{~cm}$ from the apparatus at OKC (within one body length of the apparatus). Enclosure dimensions were $5.33 \mathrm{~m}$ x $5.79 \mathrm{~m}$ (OKC Observation Enclosure), $5.18 \mathrm{~m}$ x $6.60 \mathrm{~m}$ (OKC Test Enclosure), 7.92-14.94 m x 11.28 m (NZP Observation Enclosure), and $7.92 \mathrm{~m}$ x $7.92 \mathrm{~m}$ (NZP Test Enclosure).

Table 1

Overview of Success on the Floating Object Task and Experimental Condition of Asian elephants at OKC and NZP.

\begin{tabular}{|c|c|c|c|c|c|c|}
\hline Location & $\begin{array}{l}\text { Experimental } \\
\text { Condition } \\
\end{array}$ & Subject & Sex & Age & Relationship(s) & $\begin{array}{c}\text { Solve? } \\
\text { (Y/N/T) }\end{array}$ \\
\hline \multirow{6}{*}{$\mathrm{OKC}$} & Demonstrator & Chandra & $\mathrm{F}$ & 20 & Asha's sister; Achara's aunt & $\mathrm{T}$ \\
\hline & Observer & Achara & $\mathrm{F}$ & 2 & Asha's daughter; Chandra's niece & $\mathrm{N}$ \\
\hline & Observer & Bamboo & $\mathrm{F}$ & 51 & None & $\mathrm{N}$ \\
\hline & Baseline & Asha & $\mathrm{F}$ & 21 & Achara's mother; Chandra's sister & $\mathrm{N}$ \\
\hline & Baseline & Kandula & $\mathrm{M}$ & 15 & Shanthi's son & $\mathrm{N}$ \\
\hline & Baseline & Rex & $\mathrm{M}$ & 48 & Achara's father & $\mathrm{N}$ \\
\hline \multirow{6}{*}{$\mathrm{NZP}$} & Demonstrator & Shanthi & $\mathrm{F}$ & 41 & Kandula's mother & $\mathrm{Y}$ \\
\hline & Observer & Ambika & $\mathrm{F}$ & 69 & None & $\mathrm{N}$ \\
\hline & Observer & Bozie & $\mathrm{F}$ & 42 & None & $\mathrm{N}$ \\
\hline & Observer & Swarna & $\mathrm{F}$ & 42 & None & $\mathrm{N}$ \\
\hline & Baseline & Kamala & $\mathrm{F}$ & 42 & Maharani's mother & $\mathrm{N}$ \\
\hline & Baseline & Maharani & $\mathrm{F}$ & 26 & Kamala's daughter & $\mathrm{N}$ \\
\hline
\end{tabular}

Note. The "T" indicates a trained individual. Baseline elephants that solved or were trained (Shanthi and Chandra) were subsequently treated as demonstrators for observers. 


\section{Apparatus}

The floating object tube (Mendes et al., 2007) was modified in size, durability, and trial setup for testing with elephants. The polycarbonate tube was $85.09 \mathrm{~cm}$ tall and $13.72 \mathrm{~cm}$ wide at the base, with an internal diameter of $11.12 \mathrm{~cm}$. Elephants could not fit their trunk inside of the tube, turn the tube upside down, or move the tube to reach the marshmallow. We mounted the tube on metal mesh or bars via bolts $(\mathrm{OKC})$ or ratchet straps (NZP) inside of an indoor enclosure to maximize elephants' access and minimize potential distractions (Figure 1).

\section{Test Trials}

The tube was filled about 1/3 with water. We video-recorded all trials (Sony Handycam HDRCX 405) and later extracted observational data. At the start of each trial, a keeper attracted the test subject's attention (i.e., by calling her/his name and waiting for her/him to face the keeper), presented a plain, regular-sized marshmallow (a highly preferred treat of all the elephants, and which the elephants would be motivated to retrieve compared to their other usual treats, according to keepers) (Kraft Jetpuffed Marshmallows), and dropped the marshmallow into the tube, at which point the trial timer began. We did not use peanuts as in other floating object studies, because marshmallows were the more motivating floating treat for all elephants across both institutions. All elephants had tasted a marshmallow at some point prior to testing. Elephants were locked in the enclosure during testing and were observed from out of view above the enclosure. Water was always available to subjects via an automatically refilling water drinker next to the apparatus. Trials ended when the subject solved the task or a keeper approached the elephant after ten minutes had passed. A successful solution was defined as retrieving the out-of-reach marshmallow by adding water to the apparatus and raising its water level.

Figure 1

Experimental Setup for Floating Object Demonstration Trials

A)

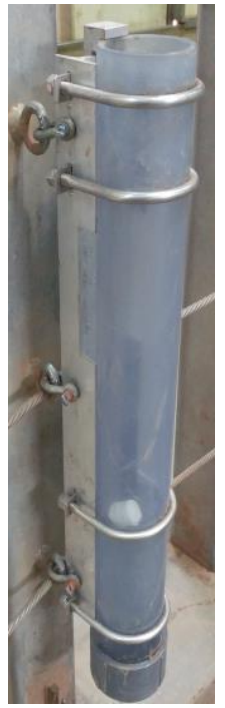

B)

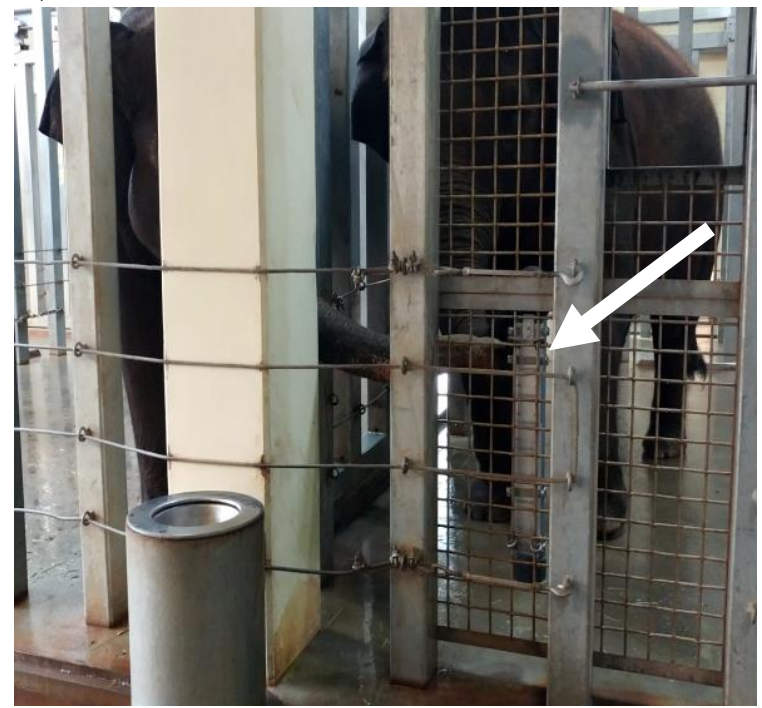

C)

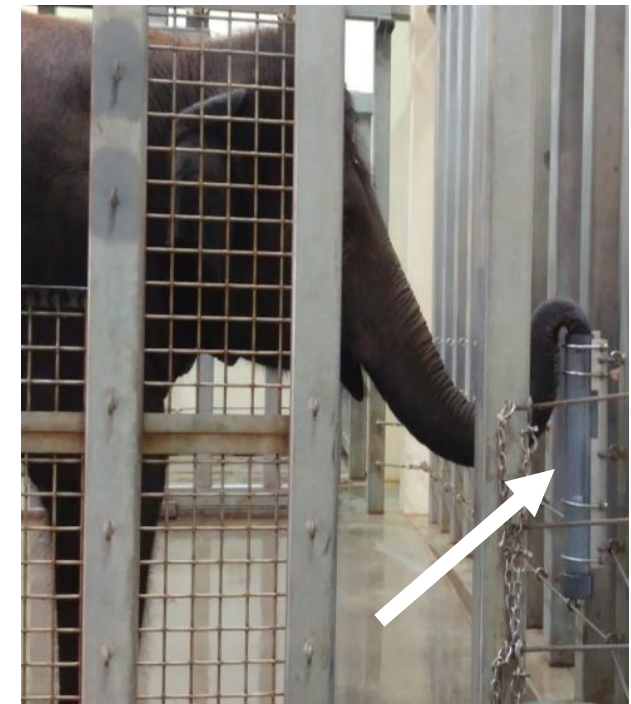

Note. Arrows indicate location of the apparatus. A) Close-up of the baited apparatus. B) A demonstrator solves the task in the right enclosure while an adjacent observer watches from the left enclosure. The observer could touch the apparatus with its trunk. C) Side-view from observer's adjacent enclosure of the demonstrator solving the task. 


\section{Demonstrators}

One female, adult elephant at each zoo, Shanthi (NZP) and Chandra (OKC), served as a demonstrator to conspecifics at her respective institution. Demonstrators were chosen based on whether they solved the task on their own (see Baseline elephants) or, if no one solved, who might be easiest to train to solve the task according to keepers (Figure 2). Demonstrators received six additional practice trials (after their individual test trials) before demonstrating for an observer.

\section{Figure 2}

Experimental Flow

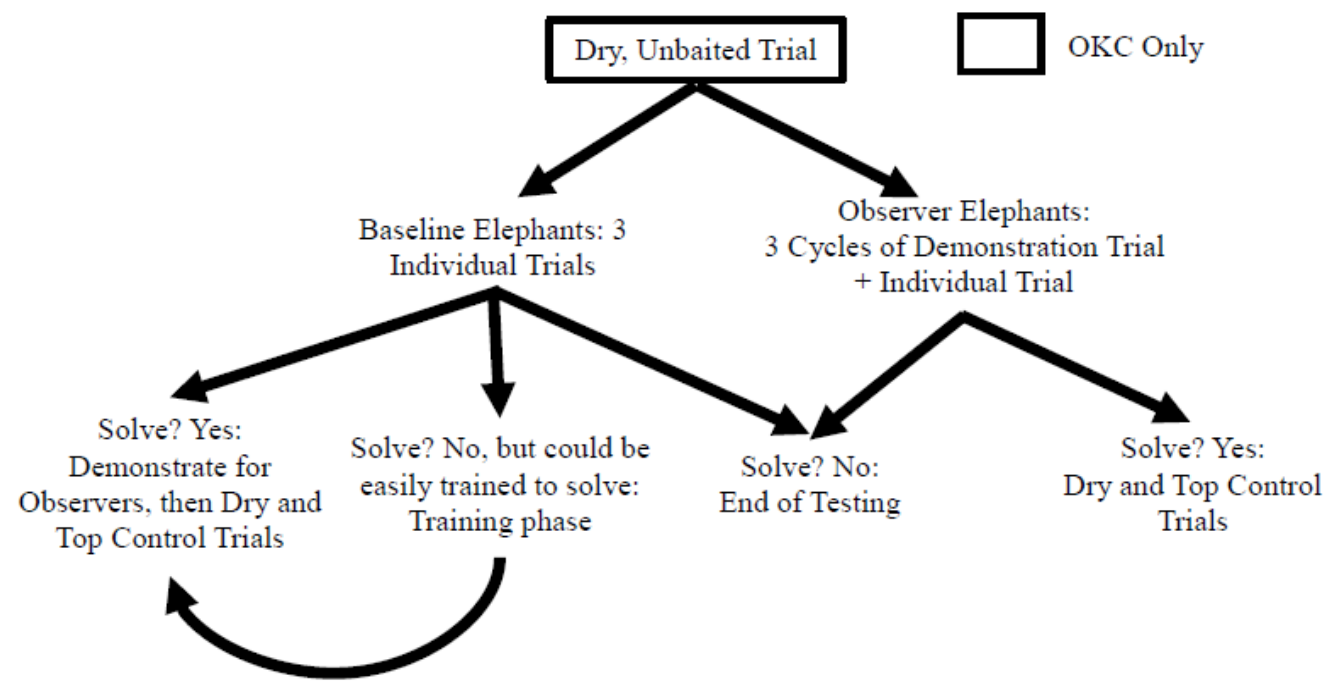

\section{Baseline Elephants}

Seven subjects (i.e., controls, or naïve individuals) received three 10-min test trials (Table 1; Figure 2). They were each presented with the task individually (i.e., did not have adjacent elephants during testing) without observing a demonstrator.

\section{Observers}

Five observers were chosen based on how well they got along with other elephants (Table 1). On each test day, observers received one demonstration from a conspecific and then one individual trial on their own for three cycles (one cycle is defined as one demonstration trial followed by one individual trial). We chose to have one demonstration before beginning individual trials based on methodology used in previous experiments investigating social learning of a novel problem-solving task (e.g., BensonAmram et al., 2014; Renner et al, 2017). Unlike previous studies, however, we were limited to three cycles due to time constraints. At the start of demonstration trials, the keeper attracted the observer's attention by calling her/his name so that the observer faced the demonstrator. The tube was not cleaned in between demonstration trials and observer test trials to preserve any chemical cues from the demonstrator. We did not allow observers and demonstrators to share the same enclosure to reduce the risk of conflict over the apparatus and reward. Observers could reach the tube and the demonstrator's water drinker from their adjacent observation enclosure. The placement of the tube was carefully planned with keepers to maximize viewing conditions for observation; we were confident that observers could see the tube and the demonstrator if they were facing the demonstrator during the demonstration (Figure 1). 


\section{Training Phase (OKC only)}

At NZP one elephant, Shanthi, solved the task on her own and was subsequently used as a demonstrator without being trained (video S1). Chandra (OKC), originally did not solve the puzzle on her own. Keepers (working with the first author) worked with Chandra to clicker-train her in three stages: (1) adding hose water to her trunk and rewarding her for adding the water to the apparatus (see video S2); (2) encouraging her to add water to her trunk from her drinking bowl and rewarding her for adding it to the apparatus (video S2; and (3) ensuring that she consistently added water to the apparatus to access the marshmallow reward on her own (i.e., at least three consecutive trials on her own and up to six additional practice trials) before proceeding to demonstration trials (video S2). The training process was fairly quick and took place across three days, for approximately 45 min each.

\section{Dry, Unbaited Trial (OKC only)}

Half of all subjects (all OKC elephants, $n=6$ ) received one trial of a dry, unbaited tube (i.e., a tube without the marshmallow) before any test trials to assess individual tendency to add water to novel items (Figure 2). This control condition had not been included in any prior floating object studies. However, following our observations at NZP, we considered that a pre-existing tendency to add water to things could explain success on the task. To test for this possibility in forthcoming trials, we added this initial task for all elephants at OKC. In this control trial, we assumed elephants would not add water to the tube, because they would not smell a food reward in the tube.

\section{Dry and Top Control Trials}

To test for further understanding of the task, after the completion of individual and demonstration trials, successful individuals were presented with a dry apparatus with a marshmallow at the bottom (i.e., subjects could not rely on having a cue of water and more water needed to be added than in regular test trials) (as in Hanus et al., 2011; Mendes et al., 2007; Renner et al., 2017). This was their penultimate trial (Figure 2). After the dry control trial, successful individuals were presented with a baited apparatus filled to the top with water (i.e., a variation of the Top control in Hanus et al. (2011) and Mendes et al. (2007) in which the tube was empty and the reward was glued to the inside of the tube). At the start of the trial, the marshmallow was floating at the top, such that no water needed to be added to the apparatus for the marshmallow to be retrieved (i.e., to see if subjects would still add water to the tube when water was not necessary). This was their final trial in the study (Figure 2). If elephants understood the requirements of the task, then we would expect them to completely fill the empty tube in their Dry control trial and to retrieve the floating marshmallow without adding any water in their Top control trial.

\section{Data Scoring and Analysis}

Video-recorded trials were coded to determine a number of outcomes related to successful problem solving on the task: latency to interact with the apparatus after the trial began, exploratory diversity (measured as the number of different behaviors exhibited toward the task; SI Table 1), time spent interacting with the apparatus, and whether each subject solved the task. To examine the impact of opportunities for social learning on problem-solving performance for observers versus baseline subjects, we used generalized linear models (GLMs) and Akaike's Information Criterion (AIC) model selection to determine if exploratory diversity, proportion of time spent interacting with the task, or latency to solve varied among observers and baseline subjects. We included location as a fixed effect to control for possible differences in testing environments (e.g., herd cohesion, distractions during testing, enclosure size and setup, etc.). To test our prediction about solvers learning and becoming more efficient over time, we used GLMs with AIC selection, using response measures latency to solve, latency to add water, and exploratory diversity as indicators of learning across trials. Demonstrators' initial three trials (i.e., before 
any training) were included with those of the baseline elephants. Data were analyzed in $R$ ( $R$ Development Core Team, 2014).

\section{Results}

\section{Dry, Unbaited Trial (OKC only)}

None of the six subjects that received the dry, unbaited trial (OKC elephants) added water to the apparatus. All subjects approached and touched the tube.

\section{Baseline Subjects and Observers}

All subjects investigated (touched) the apparatus containing a marshmallow on their first test trial. Behaviors exhibited over the course of three trials included blowing and sucking at the marshmallow and kicking the tube. Kicking did not result in any damage to the tube and subjects were not persistent with brute force methods. One baseline elephant, Shanthi (NZP), solved the task in her second and third trials (Video S1). Shanthi quickly approached and touched the tube in each trial. In her first trial, Shanthi added water once without acquiring the marshmallow. In her second test trial, Shanthi added water once, and then shot the marshmallow up out of the tube by blowing. In her third trial, Shanthi added water three times and grabbed the marshmallow. None of the five observers (of both Shanthi and Chandra) solved the task, even after receiving three demonstrations from a conspecific. No elephants had access to other objects during testing and thus could not attempt to make tools or solve using sticks or other objects.

We next compared models to determine the best predictors of proportion of time spent interacting with the task, exploratory diversity, and latency to touch. Our top model (i.e., model with the lowest AICc (Burnham \& Anderson, 2002)) for proportion of time spent interacting with the task showed that Location (NZP vs. OKC) and Condition (Baseline vs. Observer) interacted significantly (Table 2). We therefore examined all combinations of Location*Condition to determine whether observers differed from baseline elephants in proportion of time spent interacting at each zoo: At OKC, observers spent significantly more time interacting with the apparatus compared to baseline elephants (Figure 3; Post Hoc ANOVA Tukey $\mathrm{HSD}: \bar{x} \pm S D, \bar{x}_{\mathrm{OKC}}$ Baseline $\left.=0.056 \pm 0.063, \bar{x}_{\text {ОКС Observers }}=0.336 \pm 0.271, n=12, p=.031\right)$. At NZP, however, baseline elephants spent significantly more time interacting with the apparatus compared to observers (Figure 3; Post Hoc ANOVA Tukey HSD: $\overline{\mathrm{x}} \pm \mathrm{SD}, \bar{x}_{\mathrm{NZ} \text { Baseline }}=0.337 \pm 0.287, \bar{x}_{\mathrm{NZ}}$ Observers $=$ $0.071 \pm 0.118, n=12, p=.029)$. A post-hoc Tukey HSD analysis revealed that there was no difference between NZP controls and observers when we excluded Shanthi from the analysis $\left(\bar{x} \pm S D, \bar{x}_{\mathrm{NZ} \text { Baseline }}=\right.$ $0.193 \pm 0.219, \bar{x}_{\mathrm{NZ}}$ Observers $\left.=0.071 \pm 0.118, n=11, p=.494\right)$. Our top model predicting differences in latency to touch included Location (Table 2); there was a trend toward a significant difference between NZP and OKC elephants in average latency to touch $(t=1.861, n=12, p=.072)$. Exploratory diversity was also best predicted by Location (Table 2) (not Condition). There was a trend toward a significant difference between NZP and OKC elephants in their mean exploratory diversity $(z=-1.917, n=12, p=$ .055). Thus, observer and baseline elephants did not differ in their latency to touch the apparatus or their exploratory diversity, contrary to our prediction.

\section{Individual Learning}

We analyzed learning for Shanthi since she solved the task on her own. At NZP, Shanthi added some water to the tube in her first trial. Shanthi solved the puzzle in her second trial in $175 \mathrm{~s}$ and continued to solve every trial thereafter. Shanthi's latency to solve the task decreased across her individual test trials (Figure 4A; GLM: estimate $\pm S E=-33.914 \pm 7.762 \mathrm{~s}, t=-4.369, n=7$ trials, $p=$ .012 ) and latency to add water into the tube for the first time each trial decreased over trials (Figure 4B; GLM: estimate $\pm S E=-20.000 \pm 3.982 \mathrm{~s}, t=-5.023, n=7$ trials, $p=.004$ ); these findings support our prediction that if solvers learned the solution to the task, they would solve faster as trials increased and 
add water faster across trials. Shanthi's exploratory diversity did not significantly vary across her individual test trials (Figure 4C; Poisson GLM: estimate $\pm S E=-0.049 \pm 0.084 \mathrm{~s}, z=-0.583, n=7$ trials, $p$ $=.560)$, which does not support our prediction that solvers would use fewer unhelpful behaviors over time.

Table 2

Summary Table for Models Generated with $\triangle$ AICc Less Than or Equal to Two

\begin{tabular}{|c|c|c|c|c|c|c|}
\hline Model & Type (Distribution) & $d f$ & $R^{2}$ & $\mathrm{AICc}$ & $\begin{array}{c}\Delta \\
\mathrm{AICc}\end{array}$ & $\begin{array}{l}\text { Akaike } \\
\text { weight }\end{array}$ \\
\hline \multicolumn{7}{|l|}{ Latency to Touch } \\
\hline$*$ Latency to Touch $\sim$ Location & GLM (Gaussian) & 3 & 0.10 & 415.90 & 0.00 & 0.33 \\
\hline Latency to Touch $\sim$ Location + Trial Number & GLM (Gaussian) & 5 & 0.21 & 416.80 & 0.93 & 0.21 \\
\hline Latency to Touch $\sim 1$ & GLM (Gaussian) & 2 & 0.00 & 417.00 & 1.07 & 0.19 \\
\hline Latency to Touch $\sim$ Trial Number & GLM (Gaussian) & 4 & 0.13 & 417.40 & 1.55 & 0.15 \\
\hline \multicolumn{7}{|l|}{ Exploratory Diversity } \\
\hline$*$ Exploratory Diversity $\sim$ Location & GLM (Poisson) & 2 & 0.10 & 139.90 & 0.00 & 0.43 \\
\hline Exploratory Diversity $\sim$ Location + Condition & GLM (Poisson) & 3 & 0.14 & 140.70 & 0.81 & 0.29 \\
\hline Exploratory Diversity $\sim 1$ & GLM (Poisson) & 1 & 0.00 & 141.40 & 1.50 & 0.20 \\
\hline \multicolumn{7}{|l|}{ Proportion of Time Spent Interacting } \\
\hline $\begin{array}{l}* \text { Proportion of Time Spent Interacting } \sim \text { Location } \mathrm{x} \\
\text { Condition }+ \text { Location }+ \text { Condition }\end{array}$ & GLM (Beta) & 5 & 0.20 & -8.90 & 0.00 & 0.98 \\
\hline
\end{tabular}

Note. Validated models are indicated by an asterisk (*). AICc values were calculated using the "MuMIn" package in R (Barton, 2018)

Figure 3

Elephants' Proportion of Time Spent Interacting with the Floating Object Task at NZP and OKC.

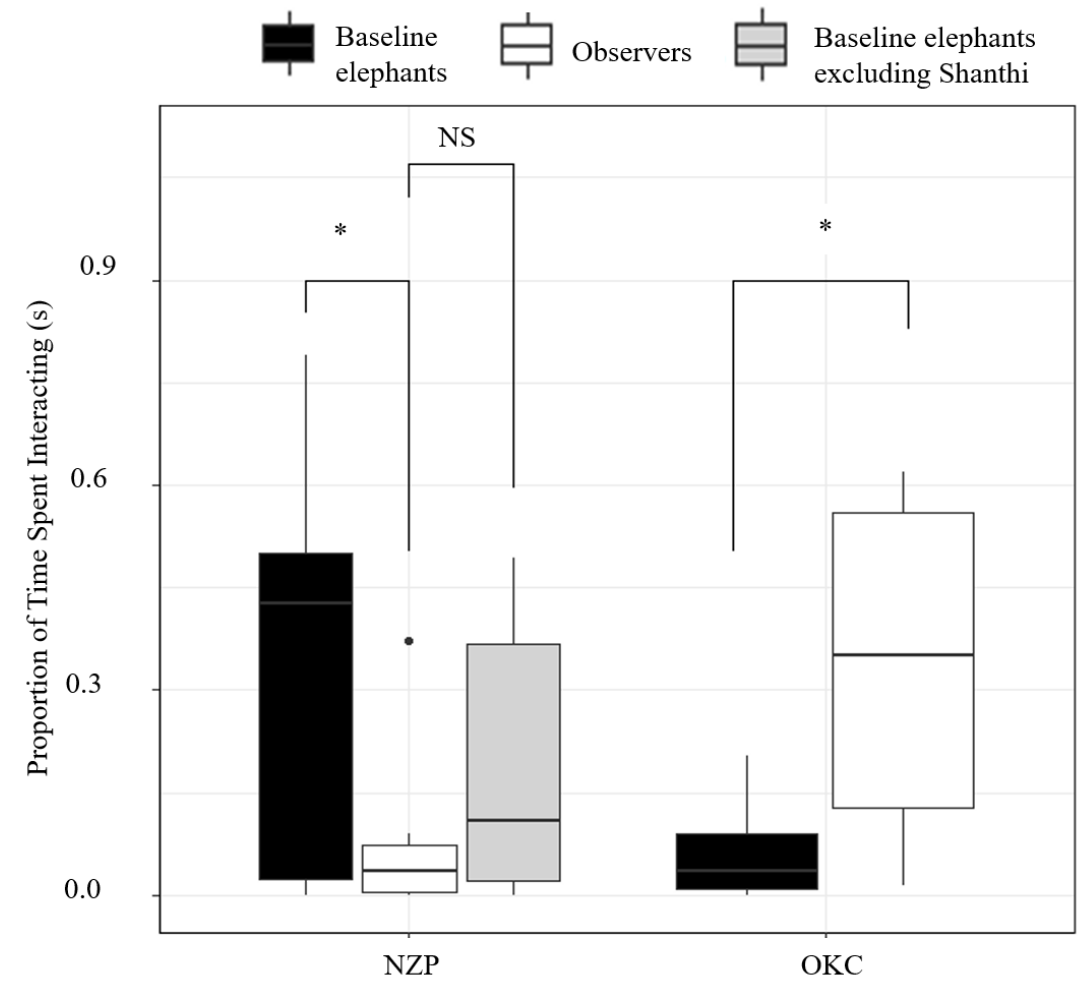




\section{Figure 4}

For NZP Baseline Subject/Demonstrator, Shanthi: A) Latency to Solve Floating Object Task Across Individual Test Trials. B) Latency to Add Water for the First Time in Each Individual Test Trial. C) Number of Different Behaviors (i.e., Exploratory Diversity) Directed Toward the Apparatus Across Individual Test Trials.

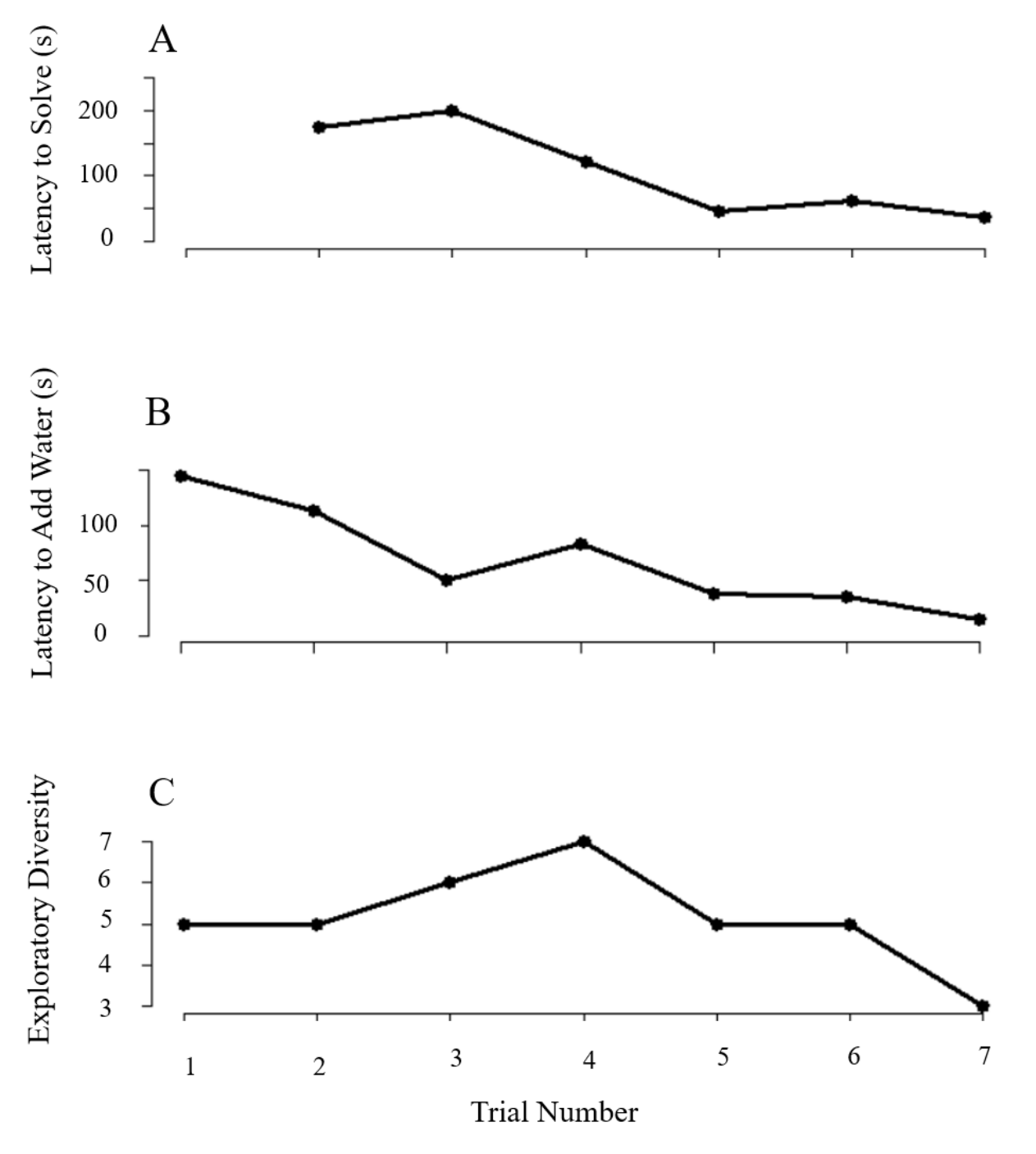

\section{Dry and Top Control Trials}

Demonstrators Shanthi and Chandra, moved on to Dry and Top control trials to test for further understanding on the task. Both Shanthi and Chandra completely filled the tube with water in their Dry control trial, as we expected if they understood the requirements of the task. They also retrieved the marshmallow floating at the top of the tube in their Top control trial. Averaged together, Shanthi and Chandra added water a greater number of times in the Dry control than in the Top control trial $\left(\bar{x}_{\text {Dry }}=5.5\right.$ times, $\bar{x}_{\text {Top }}=0.5$ times), but we could not verify this difference statistically due to our small sample size. In her Top control trial, Shanthi added one trunk-full of water to the tube after she retrieved and consumed the reward, which does not support our prediction that elephants would not add any water in the Top control trial, although our sample size was limited to one individual here. Chandra did not add water to the tube after retrieving the reward in her Top control trial, as we predicted. Shanthi and Chandra always ate the marshmallow reward after obtaining it from the tube. 


\section{Discussion}

We found that one Asian elephant (Shanthi) showed an ability to solve the floating object task without training. This supports our prediction that Asian elephants are capable of innovatively solving novel tasks. However, the low overall success rate of the Asian elephants on the floating object task demonstrates that this is a very challenging task for elephants in U.S. zoos that are trained extensively in husbandry behaviors. Shanthi's latency to solve the problem and latency to first add water gradually decreased across trials, which suggests that she learned to solve the problem via trial-and-error learning. We did not see clear evidence of insightful problem solving in this study. We also used the floating object task to investigate social learning in Asian elephants. We found evidence of stimulus enhancement, where observers increase their attention toward the apparatus toward which demonstrators showed interest, among observers at one of our study locations. Observer and baseline elephants did not differ in their latency to touch or their exploratory diversity.

To better understand the evolution of problem solving in animals, standard paradigms are implemented with a variety of species, including those from distantly related taxa and those with very different morphologies from one another (e.g., Puzzle box problem solving: carnivore species, BensonAmram et al., 2016; raccoons, Daniels et al., 2019; octopuses: Richter et al., 2016; insightful problem solving and tool use: elephants, Foerder et al., 2011; Aesop's Fable: raccoons, Stanton et al., 2017). To date, the floating object task has been used with only a limited number and diversity of species, perhaps because it was designed to assess insightful tool use and problem solving with visual species capable of containing and moving water. Here we show that, without any pre-training, one Asian elephant solved the floating object task. For comparison, in previous floating object studies conducted with more visual species, success varied when animals were given one to 10 trials and did not receive any pre-training [e.g., (successful individuals/total tested) capuchin monkeys: 0/7 (Renner et al., 2017); human adults: 14/14 (Nielsen, 2013); children: 5/36 (Nielsen, 2013) and 24/72 (Hanus et al., 2011); gorillas: 0/5 (Hanus et al., 2011); chimpanzees: 5/43 (Hanus et al., 2011) and 8/32 (Tennie et al., 2010); orangutans: 0/5 (Hanus et al., 2011) and 5/5 (Mendes et al., 2007)].

Anecdotally, the successful elephant, Shanthi, had been known to add water to enrichment items prior to the start of this study. Thus, her previous experience with water may have contributed to her success on the task. Although we implemented a dry, unbaited trial, Shanthi was not in the baseline group that received this trial. Thus, we cannot determine empirically whether she has an inherent tendency to add water to objects. Future floating object tests should aim to include dry, unbaited trials to account for a tendency to add water to enrichment, so we can confidently rule out the possibility of previous experience in performance. Importantly, the dry, unbaited control trial needs to be conducted first, before the subjects have any experience with the task.

There is currently a discussion in the literature about whether success on the floating object task can be explained by insight and causal understanding, or whether it simply reflects associative or trialand-error learning (Ghirlanda \& Lind, 2017; Hennefield et al., 2018; Shettleworth, 2012). In this study, Shanthi began solving the task in her very first trial and fully solved the task in her second trial. We cannot, however, exclude the possibility that Shanthi learned to solve the task through trial-and-error learning within a trial (or anytime previous to the present study) (e.g., via visual feedback, Ebel et al., 2019). In fact, Shanthi improved over trials, which is indicative of trial-and-error learning. Shanthi solved the Dry control trial without relying on the sight of water for solving the problem (i.e., produced the solution without seeing the required tool, Mendes et al., 2007), but at this point in the study, Shanthi had already had several trials and had experience with solving the problem. Importantly, Shanthi did not add water before retrieving the marshmallow in her Top control trial, which confirms that her behavior was goal-directed (i.e., she added water to retrieve out-of-reach food). Shanthi also continued to add water after retrieving the marshmallow in her Top control trial, perhaps due to "chaining" of previously rewarded behaviors (Bird \& Emery, 2009a; Epstein et al., 1984). It is also possible that adding water is rewarding in itself, akin to play behavior (Bird \& Emery, 2009a; raccoons interacting with stones: Stanton et al., 2017) or resulted in being able to drink equally rewarding sugary water (left from disintegrating 
marshmallow, although we never saw this behavior). Alternatively, the fact that Shanthi added water after retrieving the reward could also indicate a lack of goal-oriented behavior required for demonstrating tool use (reviewed in St. Amant \& Horton, 2008). Shanthi did not drink water from the tube after solving, so her behavior seemed to be more consistent with play behavior or an attempt to find more marshmallows. Chandra, on the other hand, did not add water after retrieving the marshmallow in her Top control trial, which could be because she quickly learned how the task worked through her training sessions. With her training sessions, Chandra had more time with the task than Shanthi overall, so perhaps with more time, Shanthi would have fully grasped the task. With so few successful elephants, we cannot conclude which cognitive abilities were involved in success on the task.

If subjects fully understand the task, we might expect that they never add water in a Top control trial. Yet subjects do occasionally add water in Top control trials of floating object studies (e.g., Hanus et al., 2011; Mendes et al., 2007), and no floating object study has explained unnecessary water-adding in Top control trials beyond a statistical comparison between subjects' frequencies of water-adding in top versus dry control trials. An empty Top control trial - in which the reward is attached to the top part of an empty tube - might be informative to see whether elephants add water when the reward is easily reachable (Hanus et al., 2011; Mendes et al., 2007). An empty Top control trial would allow us to determine whether elephants add water to tubes independently of the reward's position inside the tube and without the presence of water (Hanus et al., 2011; Mendes et al., 2007), but unfortunately we were not able to adapt this control for elephants' strength given time constraints.

We recommend that future studies of elephant cognition rely less on extractive foraging tasks, which may not be ecologically valid for elephants. A greater number of elephants may have solved the present task if it were more relevant for them, such as tasks that rely on changing the location and/or timing of resource availability (instead of using water as a tool to access food), such as a spatial or episodic memory task. That the task may have lacked ecological validity for elephants could also explain why the task may be less difficult for primates, for instance, which regularly use tools to extract food. Our study shows that the floating object task can be used for suitable species outside of primates, but unfortunately, it would have to be substantially modified (or, for example, changed to the Aesop's fable task) to be able to test other taxa that do not have the specific morphological capabilities (such as trunks) that are required for the current version of this task. The Aesop's fable task may be more suitable than the floating object task for species outside of primates because it is possible that more animals can pick up stones, or at least knock stones into a tube, than can move water. To accurately measure the evolution of cognitive abilities such as tool use and problem solving, research paradigms should be modified for testing with a wide array of species (i.e., by designing paradigms for species that rely on different sensory modalities and morphologies) — not just those that are traditionally the focus of comparative cognition.

We used a demonstrator-observer-control setup to test for social learning on the floating object task at two zoos. We took advantage of the fact that our study took place in zoos and trained one demonstrator in our social learning experiment by shaping her behavior. Observers did not solve the task, but we did find evidence of localized stimulus enhancement among observer elephants at one zoo (Hoppitt \& Laland, 2013). In other floating object tasks, emulation has been investigated and shown to occur only in chimpanzees (Tennie et al., 2010) and children (Nielsen, 2013). At NZP, baseline elephants spent more time interacting compared to observers, which we attribute to one member of the baseline group, Shanthi, solving the task. Within the constraints of the zoos, we were able to conduct only three demonstrations to observers, which may not have been sufficient for learning a novel foraging task from a conspecific. Similarly, we recommend that future studies choose more than one demonstration trial before beginning individual trials (sensu Greco et al., 2013) or use multiple demonstrators if sample sizes allow (reviewed in Hoppitt \& Laland, 2013) to give observers more opportunities to learn via social learning before any opportunity for individual trial-and-error learning, or to provide more cycles of demonstrations and test trials (e.g., Benson-Amram et al., 2014). Elephants likely prioritize smell and sound over vision in problem solving, so they may not have been watching the demonstrator. They may have instead relied on their auditory sense by hearing the sound of pouring water, and their olfactory sense by smelling the odors left by the demonstrator on the apparatus (Plotnik et al., 2014). It is possible that the auditory and 
olfactory cues were not sufficient for observer elephants to fully solve this paradigm, or that observers could not see the water level and/or the reward. Although elephants are assumed to learn socially (Bates, Poole et al., 2008; Chiyo et al., 2012; Fishlock et al., 2016; Greco et al., 2013), captive elephants - with relatively easier access to forage (they are given food in zoos) than their wild counterparts (Kummer \& Goodall, 1985) - may be less likely to rely on imitation for obtaining a relatively small food reward. In another study of Asian elephant cognition, the elephants used an experimenter's position, not visual pointing and gaze cues, in relation to food to locate the food (via local enhancement, Ketchaisri et al., 2019). Juvenile elephants learn to use their trunk and forage by interacting with related conspecifics (Lee \& Moss, 2011), yet Achara, the youngest subject, did not copy demonstrator Chandra (Achara's aunt) at OKC. Kandula exhibited insightful problem solving on another task (Foerder et al., 2011) but not in the present study, though his mother, Shanthi, did solve the present task. Unfortunately, we could not have Shanthi (NZP) demonstrate for her son, Kandula (OKC), because of their separate housing, but it would be informative to test closely related demonstrator-observer pairs (copy kin strategy: Laland, 2004). Nevertheless, more observers were related to the demonstrator at OKC compared to NZP, so this may explain the localized stimulus enhancement we observed at OKC.

In addition to relationship, demonstration quality and age differences could have factored into our findings. For example, during demonstrations, Chandra tended to solve quicker $\left(\bar{x}_{\text {Chandra }}=32.33 \mathrm{~s}, \bar{x}_{\text {Shanthi }}=\right.$ $74.89 \mathrm{~s})$ and add water a greater number of times compared to Shanthi $\left(\bar{x}_{\text {Chandra }}=3.5\right.$ times, $\bar{x}_{\text {Shanthi }}=1.89$ times), which could have affected interest of observer elephants, especially if observers had short attention spans (i.e., if they faced the demonstrator only at the start of trials and then turned away). NZP observers, in other words, had more opportunity to observe their demonstrator solving compared to OKC observers, but OKC observers saw the demonstrator add water a greater number of times than observers at NZP. Anecdotally, the OKC observers were no more attentive during demonstrations than were NZP observers. It is possible, however, that the age of the elephants contributed to whether they learned from or paid attention to the demonstrator. For instance, at $\mathrm{OKC}$, the younger Achara spent a greater amount of time interacting with the task than did Bamboo $\left(\bar{x}_{\text {Achara }}=0.44, \bar{x}_{\text {Bamboo }}=0.23\right)$. Because of our small sample size, Achara could be influencing our results, but Bamboo's proportion of time spent interacting with the task $(0.23)$ is still greater than those of the OKC baseline elephants (0.056). Furthermore, both demonstrators were younger than some of their observers. Although captive settings do not always mimic natural herd dynamics, in the wild, elephants tend to learn from an older matriarch, so it may have been unlikely for the observers to learn from the younger demonstrators in the present study. It also may have been unfair to expect that Achara - who would not have fully mastered trunk control in the same way as adults - would learn to solve this task.

It is possible that learning via training and learning via trial-and-error may have produced inherently different demonstrations by Chandra and Shanthi, respectively. For instance, if learning by training produces more rigid behavior than learning by trial-and-error, perhaps Shanthi was more variable and therefore less consistent in her demonstrations. It is also possible that there could have been other differences in their demonstrations, such as in the amounts of water they added each time. Here our design was limited by who solved the task and could serve as a demonstrator, as well as by social relationships for demonstrator-observer pairs. Future work should strive to counterbalance ages within each condition as well as use older individuals as demonstrators, with more trials for opportunities to observe. Location-specific differences, such as enclosure dimension or herd dynamics, could explain the trends we found for location differences in latency to touch and exploratory diversity. Lastly, there may have been other differences that contributed to our results. Location differences in diet, feeding times, and the apparatus' distance to the drinker, as well as individual variation in number of prior marshmallows consumed or in preference for marshmallows could have played a role in performance, for instance. 


\section{Conclusions}

In comparative cognition, the floating object task has been used as a common assessment for comparing insightful tool use across species, yet it has been used with very few taxa. Although further studies and control trials are required to determine elephants' understanding of the task, we found that at least one Asian elephant is capable of using water as a tool to solve a problem. This study provides an impetus for further testing into insightful problem solving in elephants and other non-primate, non-avian taxa.

\section{Ethical Approval}

All applicable international, national, and/or institutional guidelines for the care and use of animals were followed (National Zoological Park IACUC\# 15-25 and Oklahoma City Zoo IACUC\# 2015-013).

\section{Acknowledgments}

We are very grateful for the assistance of incredible elephant keepers and zoo staff, especially Tony Barthel, Laura Bottaro, Debbie Bastin, Rachel Emory, Debbie Flinkman, Marie Galloway, Jason Gue, Amy Hofmeister, Nick Newby, Rebecca Snyder, and Greg Vicino. Thank you especially to Karl Hanold for apparatus construction, and to research assistants Kelsea Anthony, Kendall Burnside, Haley Gordon, Jackelynn Gutierrez, Stephanie Hart, Leah Mucciarone, and Frederic Reuter for invaluable help. Many thanks to the UW Animal Behavior \& Cognition Lab, six anonymous reviewers, and Joshua Plotnik for their constructive feedback, which has greatly improved our manuscript. We acknowledge UW Biodiversity Institute, UW Zoology \& Physiology Department, and UW Botany Department for supporting this work. This material is based upon work supported by the National Science Foundation Graduate Research Fellowship under Grant No. DGE-1747504 to L.P.B.

\section{References}

Barton, K. (2018). MuMIn: Multi-Model Inference (Version 1.42.1.). R Foundation for Statistical Computing. https://doi.org/https://cran.r-project.org/package=MuMIn

Bates, L. A., Poole, J. H., \& Byrne, R. W. (2008). Elephant cognition. Current Biology, 18(13), 544-546. https://doi.org/10.1016/j.cub.2008.04.019

Bates, L. A., Sayialel, K. N., Njiraini, N. W., Moss, C. J., Poole, J. H., \& Byrne, R. W. (2007). Elephants classify human ethnic groups by odor and garment color. Current Biology, 17(22), 1938-1942. https://doi.org/10.1016/j.cub.2007.09.060

Bates, L. A., Sayialel, K. N., Njiraini, N. W., Poole, J. H., Moss, C. J., \& Byrne, R. W. (2008). African elephants have expectations about the locations of out-of-sight family members. Biology Letters, 4(1), 34-36. https://doi.org/10.1098/rsbl.2007.0529

Benson-Amram, S., Dantzer, B., Stricker, G., Swanson, E. M., \& Holekamp, K. E. (2016). Brain size predicts problem-solving ability in mammalian carnivores. Proceedings of the National Academy of Sciences, 113(9), 2532-2537. https://doi.org/10.1073/pnas.1505913113

Benson-Amram, S., Heinen, V. K., Gessner, A., Weldele, M. L., \& Holekamp, K. E. (2014). Limited social learning of a novel technical problem by spotted hyenas. Behavioral Processes, 109(PB), 111-120. https://doi.org/10.1016/j.beproc.2014.09.019

Benson-Amram, S., \& Holekamp, K. E. (2012). Innovative problem solving by wild spotted hyenas. Proceedings of the Royal Society B: Biological Sciences, 279(1744), 4087-4095. https://doi.org/10.1098/rspb.2012.1450

Bird, C. D., \& Emery, N. J. (2009a). Rooks use stones to raise the water level to reach a floating worm. Current Biology, 19(16), 1410-1414. https://doi.org/10.1016/j.cub.2009.07.033

Bird, C.D., \& Emery, N. (2009b). Insightful problem solving and creative tool modification by captive nontoolusing rooks. Proceedings of the National Acadmey of Sciences, 106(25), 10370-10375. https://doi.org/10.1073/pnas.0901008106 
Brown, B. (2018). The Conundrum of causal reasoning in elephants [unpublished master'sthesis]. University of Southern Mississippi.

Burnham, K. P., \& Anderson, D. R. (2002). Model selection and multimodal inference: A practical informationtheoretic approach (2nd ed.). Springer.

Byrne, R. W., Bates, L. A., \& Moss, C. J. (2009). Elephant cognition in primate perspective. Comparative Cognition \& Behavior Reviews, 4, 65-79. https://doi.org/10.3819/ccbr.2009.40009

Chiyo, P. I., Moss, C. J., \& Alberts, S. C. (2012). The influence of life history milestones and association networks on crop-raiding behavior in male African elephants. PLoS ONE, 7(2). https://doi.org/10.1371/journal.pone.0031382

Dale, R., \& Plotnik, J. M. (2017). Elephants know when their bodies are obstacles to success in a novel transfer task. Scientific Reports, 7(April), 1-10. https://doi.org/10.1038/srep46309

Daniels, S. E., Fanelli, R. E., Gilbert, A., \& Benson-Amram, S. (2019). Behavioral flexibility of a generalist carnivore. Animal Cognition, 22(3), 387-396. https://doi.org/10.1007/s10071-019-01252-7

de Silva, S., Ranjeewa, A. D. G., \& Kryazhimskiy, S. (2011). The dynamics of social networks among female Asian elephants. BMC Ecology, 11(1), 17. https://doi.org/10.1186/1472-6785-11-17

Duncker, K. (1945). On problem solving. Psychological Monographs, 58(5). https://doi.org/10.1037/h0093599

Ebel, S. J., Schmelz, M., Herrmann, E., \& Call, J. (2019). Innovative problem solving in great apes: The role of visual feedback in the floating peanut task. Animal Cognition, 22(5), 791-805. https://doi.org/10.1007/s10071-019-01275-0

Epstein, R., Kirshnit, C. E., Lanza, R. P., \& Rubin, L. C. (1984). "Insight" in the pigeon: Antecedents and determinants of an intelligent performance. Nature, 308(1), 61-62. https://doi.org/10.1038/309126a0

Fishlock, V., Caldwell, C., \& Lee, P. C. (2016). Elephant resource-use traditions. Animal Cognition, 19(2), 429-433. https://doi.org/10.1007/s10071-015-0921-x

Foerder, P., Galloway, M., Barthel, T., Moore, D. E., \& Reiss, D. (2011). Insightful problem solving in an Asian elephant. PLOS ONE, 6(8), e23251. https://doi.org/10.1371/journal.pone.0023251

Ghirlanda, S., \& Lind, J. (2017). 'Aesop's fable' experiments demonstrate trial-and-error learning in birds, but no causal understanding. Animal Behavior, 123, 239-247. https://doi.org/10.1016/j.anbehav.2016.10.029

Goodyear, S. E., \& Schulte, B. A. (2015). Habituation to auditory stimuli by captive African elephants (Loxodonta africana). Animal Behavior and Cognition, 2(4), 292-312. https://doi.org/10.12966/abc.11.01.2015

Gormley, R. (2015). Problem solving and tool use in three species of otter. [Master's thesis, University of Tennessee at Chattanooga]. https://scholar.utc.edu/theses/443

Greco, B. J., Brown, T. K., Andrews, J. R. M., Swaisgood, R. R., \& Caine, N. G. (2013). Social learning in captive African elephants (Loxodonta africana africana). Animal Cognition, 16(3), 459-469. https://doi.org/10.1007/s10071-012-0586-7

Hanus, D., Mendes, N., Tennie, C., \& Call, J. (2011). Comparing the performances of apes (Gorilla gorilla, Pan troglodytes, Pongo pygmaeus) and human children (Homo sapiens) in the floating peanut task. PLoS ONE, 6(6). https://doi.org/10.1371/journal.pone.0019555

Hart, B. L., \& Hart, L. A. (1994). Fly switching by Asian elephants: Tool use to control parasites. Animal Behavior, 48(1), 35-45. https://doi.org/10.1006/anbe.1994.1209

Hart, B. L., Hart, L. A., McCoy, M., \& Sarath, C. R. (2001). Cognitive behavior in Asian elephants: Use and modification of branches for fly switching. Animal Behavior, 62(5), 839-847. https://doi.org/10.1006/anbe.2001.1815

Hennefield, L., Hwang, H. G., Weston, S. J., \& Povinelli, D. J. (2018). Meta-analytic techniques reveal that corvid causal reasoning in the Aesop's Fable paradigm is driven by trial-and-error learning. Animal Cognition, 21(6), 735-748. https://doi.org/10.1007/s10071-018-1206-y

Highfill, L., Spencer, J. M., Fad, O., \& Arnold, A. M. (2016). Performance on a means-end task by Asian elephants (Elephas maximus) in a positive reinforcement-based protected-contact setting. International Journal of Comparative Psychology, 29(2).

Hoppitt, W., \& Laland, K. N. (2013). Social learning: An introduction to mechanisms, methods and models. Princeton University Press.

Irie-Sugimoto, N., Kobayashi, T., Sato, T., \& Hasegawa, T. (2008). Evidence of means-end behavior in Asian elephants (Elephas maximus). Animal Cognition, 11(2), 359-65. https://doi.org/10.1007/s10071-007-0126-Z

Jelbert, S. A., Taylor, A. H., Cheke, L. G., Clayton, N. S., \& Gray, R. D. (2014). Using the aesop's fable paradigm to investigate causal understanding of water displacement by New Caledonian crows. PLoS ONE, 9(3), e92895. https://doi.org/10.1371/journal.pone.0092895

Ketchaisri, O., Siripunkaw, C., \& Plotnik, J. M. (2019). The use of a human's location and social cues by Asian 
elephants in an object-choice task. Animal Cognition, 22(6), 907-915. https://doi.org/10.1007/s10071-019$\underline{01283-0}$

Kier, W. M., \& Smith, K. K. (1985). Tongues, tentacles and trunks: The biomechanics of movement in muscularhydrostats. Zoological Journa of the Linnean Society, 83(4), 307-324. https://doi.org/10.1111/j.10963642.1985.tb01178.x

Kummer, H., \& Goodall, J. (1985). Conditions of innovative behavior in primates. Philosophical Transactions of the Royal Society B, 308(1135), 203-214. https://doi.org/10.1098/rstb.1985.0020

Kurt, F., \& Hartl, G. B. (1995). Asian elephants (Elephas maximus) in captivity: A challenge of zoo biological research. In U. Ganslosser, J. K. Hodges, \& W. Kaumanns (Eds.), Research and captive propagation (pp. 310-326). Filander.

Laland, K. N. (2004). Social learning strategies. Learning \& Behavior, 32(1), 4-14. https://doi.org/10.3758/BF03196002

Lee, P. C., \& Moss, C. J. (2011). Calf development and maternal rearing strategies. In C. J.Moss, , H. Croze, \& P. C. Lee (Eds.), The Amboseli elephants: A long-term perspective on a long-lived mammal (pp. 224-237). The University of Chicago Press.

Logan, C. J. (2016). Behavioral flexibility and problem solving in an invasive bird. PeerJ, 4:e1975. https://doi.org/10.7717/peerj.1975

Logan, C. J., Jelbert, S. A., Breen, A. J., Gray, R. D., \& Taylor, A. H. (2014). Modifications to the Aesop's fable paradigm change New Caledonian crow perofrmances. PLoS ONE, 9(7), 1-10. https://doi.org/10.1371/journal.pone.0103049

McComb, K., Moss, C., Durant, S. M., Baker, L., \& Sayialel, S. (2001). Matriarchs act as repositories of social knowledge in African elephants. Science, 292(5516), 491-494. https://doi.org/10.1126/science.1057895

McComb, K., Moss, C., Sayialel, S., \& Baker, L. (2000). Unusually extensive networks of vocal recognition in African elephants. Animal Behavior, 59(6), 1103-1109. https://doi.org/10.1006/anbe.2000.1406

McComb, K., Shannon, G., Durant, S. M., Sayialel, K., Slotow, R., Poole, J., \& Moss, C. (2011). Leadership in elephants: The adaptive value of age. Proceedings of the Royal Society B: Biological Sciences, 278(1722), 3270-3276. https://doi.org/10.1098/rspb.2011.0168

McComb, K., Shannon, G., Sayialel, K. N., \& Moss, C. (2014). Elephants can determine ethnicity, gender, and age from acoustic cues in human voices. Proceedings of the National Academy of Sciences of the United States of America, 111(14), 5433-5438. https://doi.org/10.1073/pnas.1321543111

McKay, G. (1973). Behavior and ecology of the Asiatic elephant in southeastern Ceylon. Smithsonian Contributions to Zoology, 125, 1-113. https://doi.org/10.5479/si.00810282.125

Mendes, N., Hanus, D., \& Call, J. (2007). Raising the level: Orangutans use water as a tool. Biology Letters, 3(5), 453-455. https://doi.org/10.1098/rsbl.2007.0198

Mizuno, K., Irie, N., Hiraiwa-Hasegawa, M., \& Kutsukake, N. (2016). Asian elephants acquire inaccessible food by blowing. Animal Cognition, 19(1), 215-222. https://doi.org/10.1007/s10071-015-0929-2

Moss, C. (1988). Elephant memories: Thirteen years in the life of an elephant family. William Morrow.

Nielsen, M. (2013). Young children's imitative and innovative behavior on the floating object task. Infant and Child Development, 22(1), 44-52. https://doi.org/10.1002/icd.1765

Nissani, M. (2006). Do Asian elephants (Elephas maximus) apply causal reasoning to tool-use tasks? Journal of Experimental Psychology: Animal Behavior Processes, 32(1), 91-96. https://doi.org/10.1037/00977403.32.1.91

Pettigrew, J. D., Bhagwandin, A., Haagensen, M., \& Manger, P. R. (2010). Visual acuity and heterogeneities of retinal ganglion cell densities and the tapetum lucidum of the African elephant (Loxodonta africana). Brain, Behavior and Evolution, 75(4), 251-261. https://doi.org/10.1159/000314898

Plotnik, J.M., Brubaker, D.L., Dale, R., Tiller, L.N., Mumby, H.S., \& Clayton, N.S. (2019). Elephants have a nose for quantity. Proceedings of National Academy of Sciences, 116(25), 12566-12571. https://doi.org/10.1073/pnas.1818284116

Plotnik, J. M., \& de Waal, F. B. M. (2014a). Asian elephants (Elephas maximus) reassure others in distress. PeerJ, 2, e278. https://doi.org/10.7717/peerj.278

Plotnik, J. M., \& de Waal, F. B. M. (2014b). Extraordinary elephant perception. Proceedings of the National Academy of Sciences of the United States of America, 111(14), 10-11. https://doi.org/10.1073/pnas.1403064111

Plotnik, J. M., de Waal, F. B. M., Moore, D., \& Reiss, D. (2010). Self-recognition in the Asian elephant and future directions for cognitive research with elephants in zoological settings. Zoo Biology, 29(2), 179-191. https://doi.org/10.1002/zoo.20257 
Plotnik, J. M., de Waal, F. B. M., \& Reiss, D. (2006). Self-recognition in an Asian elephant. Proceedings of the National Academy of Sciences of the United States of America, 103(45), 17053-17057. https://doi.org/10.1073/pnas.0608062103

Plotnik, J. M., Lair, R., Suphachoksahakun, W., \& de Waal, F. B. M. (2011). Elephants know when they need a helping trunk in a cooperative task. Proceedings of the National Academy of Sciences, 108(12), 5116-5121. https://doi.org/1101765108 [pii]ไr10.1073/pnas.1101765108

Plotnik, J. M., Shaw, R. C., Brubaker, D. L., Tiller, L. N., \& Clayton, N. S. (2014). Thinking with their trunks: Elephants use smell but not sound to locate food and exclude nonrewarding alternatives. Animal Behavior, 88, 91-98. https://doi.org/10.1016/j.anbehav.2013.11.011

Polla, E. J., Grueter, C. C., \& Smith, C. L. (2018). Asian elephants (Elephas maximus) discriminate between familiar and unfamiliar human visual and olfactory cues. Animal Behavior and Cognition, 5(3), 279-291. https://doi.org/10.26451/abc.05.03.03.2018

Povinelli, D. J. (2000). Folk physics for apes: The chimpanzee's theory of how the world works. Oxford University Press.

R Development Core Team. (2014). R: A language and environment for statistical computing. R Foundation for Statistical Computing,Vienna, Austria. https://www.R-project.org/

Renner, E., Abramo, A. M., Karen Hambright, M., \& Phillips, K. A. (2017). Insightful problem solving and emulation in brown capuchin monkeys. Animal Cognition, 20(3), 531-536. https://doi.org/10.1007/s10071017-1080-Z

Richter, J. N., Hochner, B., \& Kuba, M. J. (2016). Pull or push? Octopuses solve a puzzle problem. PLoS ONE, 11(3), 1-16. https://doi.org/10.1371/journal.pone.0152048

Shettleworth, S. J. (2010). Cognition, evolution, and behavior (2nd ed.). Oxford University Press.

Shettleworth, S. J. (2012). Do animals have insight, and what is insight anyway? Canadian Journal of Experimental Psychology, 66(4), 217-226. https://doi.org/10.1037/a0030674

Shoshani, J., Kupsky, W. J., \& Marchant, G. H. (2006). Elephant brain. Part I: Gross morphology, functions, comparative anatomy, and evolution. Brain Research Bulletin, 70(2), 124-157. https://doi.org/10.1016/j.brainresbull.2006.03.016

Smith, K. K., \& Kier, W. M. (1989). Trunks, tongues, and tentacles: Moving with skeletons of muscle. American Scientist, 77(1), 28-35.

St. Amant, R., \& Horton, T. E. (2008). Revisitng the definition of animal tool use. Animal Behavior, 75(4), 11991208. https://doi.org/10.1016/j.anbehav.2007.09.028

Stanton, L., Davis, E., Johnson, S., Gilbert, A., \& Benson-Amram, S. (2017). Adaptation of the Aesop's Fable paradigm for use with raccoons (Procyon lotor): Considerations for future application in non-avian and non-primate species. Animal Cognition, 20(6), 1147-1152. https://doi.org/10.1007/s10071-017-1129-z

Stone, J., \& Halasz, P. (1989). Topography of the retina in the elephant Loxodonta africana. Brain, Behavior and Evolution, 34(2), 84-95. https://doi.org/10.1159/000116494

Taylor, A. H., Elliffe, D. M., Hunt, G. R., Emery, N. J., Clayton, N. S., \& Gray, R. D. (2011). New Caledonian crows learn the functional properties of novel tool types. PLoS ONE, 6(12), e26887. https://doi.org/10.1371/journal.pone.0026887

Tennie, C., Call, J., \& Tomasello, M. (2010). Evidence for emulation in chimpanzees in social settings using the floating peanut task. PLoS ONE, 5(5), e10644. https://doi.org/10.1371/journal.pone.0010544

Thorpe, W. H. (1963). Learning and instinct in animals (2nd ed.). Methuen.

Veling, H., \& Aarts, H. (2012). Routinized learning of behavior. In N. M. Seel (Ed.), Encyclopedia of the sciences of learning (pp. 2905-2907). Springer. https://doi.org/10.1007/978-1-4419-1428-6

Wickler, W., \& Seibt, U. (1997). Aimed object-throwing by a wild African elephant in an interspecific encounter. Ethology, 103(5), 365-368. https://doi.org/10.1111/j.1439-0310.1997.tb00152.x

Yokoyama, S., Takenaka, N., Agnew, D. W., \& Shoshani, J. (2005). Elephants and human color-blind deuteranopes have identical sets of visual pigments. Genetics, 170(1), 335-344. https://doi.org/10.1534/genetics.104.039511

Zajonc, R. B. (1965). Social facilitation. Science, 149(3681), 269-274. https://doi.org/10.1126/science.149.3681.269 